\title{
Proteomic identification of potential markers of myosteatosis in human urine
}

\author{
HOLGER HUSI $^{1}$, ALISDAIR MacDONALD ${ }^{2}$, RICHARD J.E. SKIPWORTH ${ }^{2}$, JANICE MILLER ${ }^{2}$, \\ ANDREW CRONSHAW ${ }^{3}$, KENNETH C.H. FEARON ${ }^{2 *}$ and JAMES A. ROSS ${ }^{2}$ \\ ${ }^{1}$ Department of Diabetes and Cardiovascular Science, University of the Highlands and Islands, \\ Centre for Health Science, IV2 3JH Inverness; ${ }^{2}$ Department of Clinical Sciences, University of Edinburgh, \\ EH16 4SB Edinburgh; ${ }^{3}$ School of Biological Sciences, University of Edinburgh, Edinburgh, UK
}

Received March 7, 2018; Accepted April 19, 2018

DOI: 10.3892/br.2018.1091

\begin{abstract}
Myosteatosis, the infiltration of fat in skeletal muscle, is associated with lower skeletal muscle density (SMD) as detected by computed tomography (CT). It increases with aging and obesity and is thought to play a role in the aetiology of insulin resistance and type II diabetes. The clinical significance of myosteatosis in cancer cachexia, however, remains to be determined. Along with demonstrable subcutaneous and visceral lipolysis, myosteatosis may also be a key component of the syndrome. We aimed to investigate the use of human urine as a non-invasive way to screen for molecular biomarkers of myosteatosis/reduced SMD using SELDI-TOF mass spectrometry. Pre-operative CT scans of patients undergoing surgery for upper gastrointestinal or hepatopancreaticobiliary cancer were analysed at the level of the third lumbar vertebrae. Myosteatosis was inferred as the presence of reduced SMD, which was defined as Hounsfield units for skeletal muscle $<39.5$ (two standard deviations below a normal healthy cohort). Urine was analysed by mass spectrometry using CM10 and IMAC30 SELDI-chips. Peaks observed in the CM10 and IMAC30 chip types, showed marked expressional differences between control and myosteatosis, were further investigated by mascot SELDI matrix matching. A total of 55 patients was recruited; 31 patients were found to be
\end{abstract}

Correspondence to: Professor James A. Ross, Department of Clinical Sciences, University of Edinburgh, Room FU501, Chancellors Building, 49 Little France Crescent, EH16 4SB Edinburgh, UK

E-mail: j.a.ross@ed.ac.uk

\section{"Deceased}

Abbreviations: BPS, Biomarker Pattern software; emPAI, exponentially modified protein abundance index; uGI, upper gastrointestinal; OGJ, oesophagogastric junction; PCDMS, ProteinChip Data Manager software; SPA, sinapinic acid

Key words: SELDI-TOF, myosteatosis, muscle wasting, urine biomarker myosteatotic on CT scan. Application of the IMAC30-derived model to the entire cohort showed a sensitivity of $97 \%$, specificity of $71 \%$ and an overall correctness of $85 \%$. Application of the CM10 chipset-based model to the entire cohort, showed a $77 \%$ sensitivity, $67 \%$ specificity and $73 \%$ overall correctness. Analysis of the peaks of interest resulted in the identification of significant fragments of cathepsin C, argin, arylsulfatase A and glial fibrillary acidic protein. We identified several potential urinary molecular biomarkers associated with reduced SMD in cancer. Such markers are potentially useful in deriving a clinical screening test for myosteatosis.

\section{Introduction}

Myosteatosis occurs in two forms: Fat deposition within myocytes (intramyocellular fat) and fat infiltration within the fascia between muscle layers (intermuscular fat) (1), although the latter is excluded by computed tomography (CT) scan analysis. Myosteatosis increases with aging and obesity, but it may also play a role in the development of human disease (1). The clinical significance of myosteatosis remains unclear but it appears to be involved in the aetiology of insulin resistance and type II diabetes mellitus through the modulation of both adipokine (e.g., adiponectin) secretion and nutritive muscle blood flow (2).

Cancer cachexia is a severe syndrome of skeletal muscle wasting and lipolysis that appears to develop as part of an aberrant host response to the presence of tumour (3). The clinical consequences of cachexia include increased rates of patient morbidity and mortality, and decreased rates of oncologic response to chemotherapy $(4,5)$. Despite demonstrable subcutaneous and visceral lipolysis during cancer cachexia, myosteatosis and sequestration of fat into skeletal muscle may be a key component of the syndrome (6). Several pro-inflammatory cytokines (e.g., IL- 6 and TNF- $\alpha$ ) and adipokines [e.g., zinc- $\alpha 2$-glycoprotein (ZAG) are involved in the aetiology of cancer cachexia] $(7,8)$. In the past, members of our group have identified increased mRNA expression of ZAG a proposed lipid mobilising factor, in fat samples from cachectic cancer patients; however, serum ZAG levels were unchanged from controls (9). Visceral adipose tissue is lost more rapidly than subcutaneous adipose tissue during cachexia, suggesting 
differential tissue-dependent responses to the wasting process. Recently, focus has concentrated on the concept of 'fat-muscle crosstalk' in cancer cachexia. Notably, the genetic ablation of lipolytic pathways in adipocytes protects against muscle mass loss in pre-clinical models of cancer cachexia (10). These data support the loss of visceral fat driven by lipolytic mechanisms as an early event in cancer cachexia with a potential effect on skeletal muscle.

We previously used cross-sectional magnetic resonance imaging (MRI) of quadriceps to demonstrate increased intermuscular fat in cancer patients compared with both elderly and young healthy controls (11). We also combined cross-sectional CT at the level of the third lumbar vertebra with ultra-structural analysis of rectus abdominis muscle biopsies by electron microscopy to demonstrate that intramyocellular lipid deposition is increased in cancer patients compared with controls (12). However, whether myosteatosis is a cause or effect of cancer cachexia remains to be elucidated. Certainly, insulin resistance is a key finding in previous clinical studies of cancer cachexia, with cancer patients demonstrating lower serum insulin and insulin: cortisol ratios, and higher serum glucose (13). Such insulin resistance represents one causative mechanism of skeletal muscle protein depletion in cancer cachexia through a lack of anabolic phosphatidylinositol 3 kinase pathway signalling (13). Myosteatosis may play an even greater role in the development of cancer cachexia in those patients with obesity in the presence of skeletal muscle depletion, so-called 'sarcopenic obesity'. Previous cross-sectional CT studies showed that it is these individuals that have the worst overall prognosis from advanced lung and pancreatic cancer (14).

In an attempt to identify diagnostic biomarkers of cancer cachexia, we previously showed that MALDI-TOF mass spectrometric (MS) analysis can identify breakdown products of skeletal muscle protein in human urine (15). Furthermore, SELDI-TOF MS was capable of identifying diagnostic markers of upper gastrointestinal (uGI) cancer in the same substrate (16). Therefore, we hypothesised that a SELDI-TOF platform would also be used to identify protein/peptide biomarkers of myosteatosis in the urine of patients with cancer. The identity of such markers would not only offer insights into the development of myosteatosis, but also identify high-risk groups among individuals with cancer-associated weight loss.

\section{Materials and methods}

Materials. Buffers, gels and SELDI chips were from Bio-Rad (Hemel Hempstead, UK), and all other chemicals were obtained from Sigma-Aldrich (Gillingham, UK) unless stated otherwise in the text.

Sample collection. Urine samples were obtained from uGI and hepatopancreaticobiliary (HPB) cancer patients who were undergoing potentially curative surgery approximately 4-6 weeks after the cessation of chemotherapy. Urine samples were collected into a sterile container at induction of anaesthesia. They were snap frozen in liquid nitrogen and stored at $-80^{\circ} \mathrm{C}$ until use. The procedures were approved by the local research ethics committee. Written informed consent was obtained.
The study conformed to the standards set by the Declaration of Helsinki.

Myosteatosis stratification. Skeletal muscle CSA was measured from routine CT scans performed prior to any surgical or oncological intervention. CT scans were recorded within an average of 23 days prior to supplying the urine samples. Digitally stored CT images completed with a spiral CT scanner for initial staging and routine diagnostic purposes were analysed using semi-automated software that permitted specific tissue demarcation using Hounsfield unit thresholds of -29 to +150 for skeletal muscles, -150 to -50 for visceral adipose tissue, and -190 to -30 for subcutaneous adipose tissue. Cross-sectional areas $\left(\mathrm{cm}^{2}\right)$ were computed for each tissue by summing tissue pixels and multiplying by the pixel surface area. A transverse CT image from the third lumbar vertebrae (L3) was assessed for each scan date and tissue volumes estimated. The muscles in the L3 region contain psoas, erector spinae, quadratus lumborum, transversus abdominus, external and internal oblique, and rectus abdominis. CT images were analysed by a single trained observer, tissue boundaries were manually corrected as needed. Cross-sectional area for muscle was normalized for stature $\left(\mathrm{cm}^{2} / \mathrm{m}^{2}\right)$. Cut-off values for low muscularity were based on a CT-based sarcopenic obesity study of cancer patients (i.e., L3 skeletal muscle index: $\leq 38.5 \mathrm{~cm}^{2} / \mathrm{m}^{2}$ for women and $\leq 52.4 \mathrm{~cm}^{2} / \mathrm{m}^{2}$ for men) (17) Myosteatosis was defined by the mean Hounsfield units for skeletal muscle, and values $<39.5$ (two standard deviations below a normal healthy cohort) were regarded as myosteatotic.

SELDI-TOF-MS. SELDI-chips (CM10 and IMAC30) were prepared for sample application according to the manufacturer's recommendations (16). IMAC30 chips were loaded with $0.1 \mathrm{M} \mathrm{CuSO}_{4}$ and washed with water. Chips were neutralised with 0.1 M NaHAc ( $\mathrm{pH} 4.0$ ) and washed with water. A further 2 washes with $0.1 \mathrm{M} \mathrm{NaHPO}_{4}$ were completed. CM10 chips and $0.5 \mathrm{M} \mathrm{NaCl}$ were washed twice with $0.1 \mathrm{M} \mathrm{NaHPO}_{4}$ ( $\mathrm{pH} 4.0$ ). All chips were processed in a bioprocessor-assembly by incubating $0.1 \mathrm{ml}$ urine and $0.1 \mathrm{ml}$ binding buffer [CM10: 0.1 M NaHPO $(\mathrm{pH}$ 4.0); IMAC30: 0.1 M NaHPO $0.5 \mathrm{M} \mathrm{NaCl}$ ] for $1 \mathrm{~h}$ at room temperature. Three washes were completed with $0.2 \mathrm{ml}$ binding buffer for $5 \mathrm{~min}$ each at room temperature and 2 washes with $0.2 \mathrm{ml}$ water. Samples were air dried and 2 times $1 \mathrm{ml}$ energy absorbing matrix [sinapinic acid (SPA) in 50\% CAN, 0.5\% TFA] was added. Air-dried chips were analysed in PCS4000 SELDI-TOF instrument (Bio-Rad) by measuring the 1,000 to 25,000 Da range with a laser setting of $2.5 \mathrm{~mJ}$. Spectra was exported as '.xml' files. The SELDI instrument was calibrated using the ProteinCip All-In-one peptide standard (Bio-Rad). Source voltage was set as $25,000 \mathrm{~V}$ and detector voltage as 2,946 V. Quality control and consistency was ensured by using one random pool of urines on one spot per chip each. Spectral alignments of all quality controls ensured consistency of all spectra.

Data processing. ProteinChip Data Manager software (PCDMS) version 4.1 with integrated Biomarker Wizard cluster analysis (Bio-Rad) was used for analysis. SELDI-TOF-MS traces were split into two groups: Control and myosteatosis based on CT scan analysis. The baseline was subtracted from 
individual $\mathrm{m} / \mathrm{z}$ traces and profiles were normalised using total ion current, followed by identification of peak clusters using the cluster analysis tool. Peaks were selected in the first pass where the signal to noise $(\mathrm{S} / \mathrm{N})$ ratio was $>5$ and had a valley depth of at least 3 . In the second pass the $\mathrm{S} / \mathrm{N}$ ratio was 2 and the valley depth was also 2 . The cluster mass window was set to $0.2 \%$ of mass. Clustered peaks were only included if they occurred in at least $10 \%$ of all spectra. The resulting P-values, ROC areas, mean and median $\mathrm{m} / \mathrm{z}$ values, and intensities of the clustered peaks were exported and saved as '.csv' files and used for model building. Heat-maps using Pearson's correlation and principle component analysis plots were calculated to assess global group divisions (i.e., myosteatosic and non-myosteatotic). A two-sample t-test was used to compare mean normalised intensities between the case and control groups. $\mathrm{P}<0.05$ was considered statistically significant.

Model building and validation. Clustered peak lists were analysed with the Biomarker Pattern software (BPS; Bio-Rad). The $\mathrm{m} / \mathrm{z}$ versus intensity matrices were analysed using decision tree-analysis, selecting the standard error rule of minimum cost-tree regardless of size. The method used was Gini. V-fold testing was set to 1,000 . A total of 20 myosteatosis samples and 20 control samples were chosen randomly and used as the learning and testing dataset. The remaining 15 samples were used as the validation dataset for blind-testing. Sensitivity was defined as the probability of predicting myosteatosis cases, and the specificity was defined as the probability of predicting non-myosteatotic samples.

Peak isolation and identification. Peaks observed in the CM10 and IMAC30 chip-types which show marked expression differences between control and myosteatosis samples, or were branching points in the models were further investigated as detailed below: $0.5 \mathrm{ml}$ of urine from positive or negative samples in relation to specific peaks was added to $30 \mathrm{ml} \mathrm{CM10}$ or IMAC30 spin column resins (Bio-Rad). A total of $0.75 \mathrm{ml}$ of binding buffer [0.1 $\mathrm{M} \mathrm{NaHPO}_{4}(\mathrm{pH} 4.0)$ for CM10 resins and $0.1 \mathrm{M} \mathrm{NaHPO}_{4}(\mathrm{pH} 7.0)$ and $0.5 \mathrm{M} \mathrm{NaCl}$ for IMAC30 resins]was then added. These were incubated for $1 \mathrm{~h}$ at room temperature under constant agitation. Unbound material was removed and the resin was washed 4 times with $0.3 \mathrm{ml}$ binding buffer. Bound material was separated by electrophoresis on a $16.5 \%$ Tris-Tricine gel (Bio-Rad) and gel bands in the region of 2 to $10 \mathrm{kDa}$ were excised after Coomassie staining (BioSafe Coomassie; Bio-Rad). Positive and negative samples were chosen on the presence or absence of a specific $\mathrm{m} / \mathrm{z}$ peak to be identified based on SELDI-TOF-MS analysis. Proteins and peptides from gel bands were digested in situ with trypsin, the resulting peptides eluted with $\mathrm{ACN}$, and analysed using LC-MS/MS as previously described (16). Fragmentation spectra were then processed by Xcalibur and BioWorks software (Thermo Fisher Scientific, Loughborough, UK) and submitted to the Mascot search engine (Matrix Science, London, UK) using UniProt/SwissProt (release July 2010 , Homo sapiens, 18,055 sequences) as the reference database. Mascot search parameters were: Enzyme specificity trypsin, maximum missed cleavage 1 , fixed modifications cysteine carbamidomethylation, variable modification methionine oxidation, precursor mass tolerance +/-3 Da, fragment ion mass tolerance +/-0.4 Da. Only Mascot hits with a false discovery rate of $<0.05$ were taken into consideration. Proteins with $\geq 2$ peptide matches were analysed by pattern matching based on SELDI-TOF-MS measured expression levels of peaks of interest (expected abundance in selected samples) and observed presence of proteins. Peptide distribution of identified peptides within a protein as well as calculated molecular mass of identified proteins was used to assess whether breakdown products were likely to account for mass variances between the expected mass and the molecular weight of the full-length protein.

Mascot-SELDI matrix matching. Observed proteins with at least two peptide matches from the LC-MS/MS analysis were further analysed by pattern matching based on SELDI-TOF-MS measured expression levels of peaks of interest (expected abundance in selected samples). This was carried out using software written in-house, which compared observed protein expression patterns in a pre-defined set of samples (LC-MS/MS results) against a matrix of peak patterns (SELDI-TOF clustered peak intensities, where estimated peaks are set to null) in the same set of samples. Scoring was based on sensitivity (percent observed over expected) and specificity (percent not observed over not expected). Results are presented in descending order of cumulative scores.

\section{Results}

Patient data. A total of 55 patients (mean age, 64 years) with uGI cancer was stratified according to the presence or absence of myosteatosis with 24 being regarded as non-myosteatotic (Table I). Data variables, with the exception of plasma CRP, had normal distributions. The non-myosteatotic control group was significantly shorter, lighter and older than the Myosteatotic group but had similar BMI and weight loss (Student's t-test) and similar CRP (Mann-Whitney U).

Four peak clusters in the IMAC30, and 8 in the CM10 datasets showed statistically significant P-values $<0.05$, which indicates a potentially significant difference in the expression levels for a particular protein or peptide cluster.

Decision tree analysis. Decision-tree modelling using the BPS of peak clusters of 20 random samples of each cohort was validated by the remainder of the entire cohort 4 control and 11 myosteatotic samples). Decision tree models are shown in Fig. 1A and D. The IMAC30 chipset-based model (Fig. 1A) showed a sensitivity of $100 \%$ and a specificity of $25 \%$ for the validation dataset, and application of the derived model to the entire cohort showed a sensitivity of $97 \%$, and specificity of $71 \%$. Application of the CM10 chipset-based model (Fig. 1D) to the validation dataset gave a sensitivity of $73 \%$, specificity of $25 \%$, and for the entire cohort the values are $77 \%$ sensitivity, $67 \%$ specificity.

Statistical analysis of the $\mathrm{m} / \mathrm{z}$ cluster peaks involved in the models and potential marker peaks showing low P-values are shown in Table II.

Of the 4 peak clusters in the IMAC30-based dataset which had either high model score values, or showed P-values $<0.05$, three were upregulated and one downregulated in myosteatosis (e.g., m/z 2,540 in Fig. $1 \mathrm{~B}$ and m/z 4,373 in Fig. 1). 
Table I. Demographics of the cohort $(n=55)$.

\begin{tabular}{lccc}
\hline Characteristics & Non-myosteatotic, $\mathrm{n}=24$ & Myosteatosis, $\mathrm{n}=31$ & Entire cohort, $\mathrm{n}=55$ \\
\hline Mean age (years) & $60(10)$ & $67(7)^{\mathrm{a}}$ & $64(9)$ \\
Male:female & $18: 6$ & $27: 4$ & $45: 10$ \\
Height $\left(\mathrm{cm}^{2}\right)$ & $168.7(8.4)$ & $174.8(7.3)^{\mathrm{a}}$ & $172.1(8.3)$ \\
Weight $(\mathrm{kg})$ & $72.7(16.5)$ & $82.9(14.9)^{\mathrm{a}}$ & $78.4(16.3)$ \\
Weight loss $(\%)$ & $6.7(8.8)$ & $5.3(6.9)$ & $5.9(7.7)$ \\
BMI & $25.5(4.7)$ & $27.2(4.6)$ & $26.4(4.7)$ \\
CRP (mg/l) & $13.5(34.0)$ & $12.5(31.9)$ & $12.9(32.5)$ \\
Skeletal muscle mass & $47.8(9.9)$ & $46.2(7.6)$ & $46.9(8.6)$ \\
Mean HU & $45.9(4.6)$ & $33.6(4.6)^{\mathrm{a}}$ & $39.0(7.7)$ \\
\hline
\end{tabular}

Data is mean \pm standard deviation or median (range) for $\mathrm{C}$-reactive protein $(\mathrm{CRP})$. ${ }^{\mathrm{a}} \mathrm{P}<0.05$, myosteatosis vs. non-myosteatosis (Student's $\mathrm{t}$-test, Mann-Whitney U). BMI, body mass index.

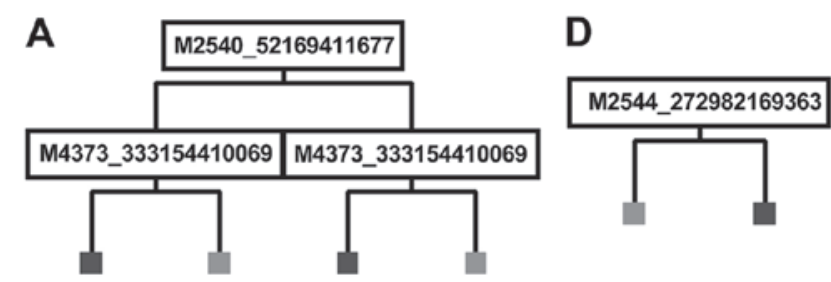

B

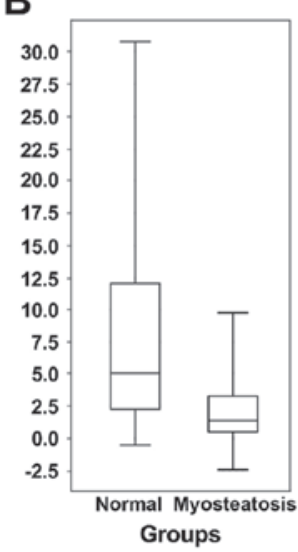

C

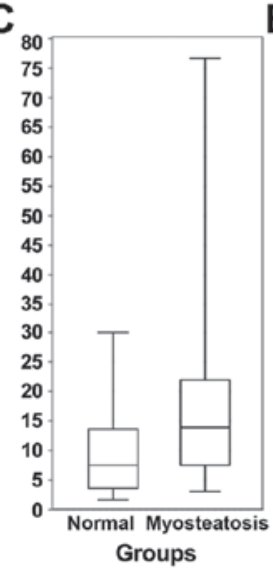

E

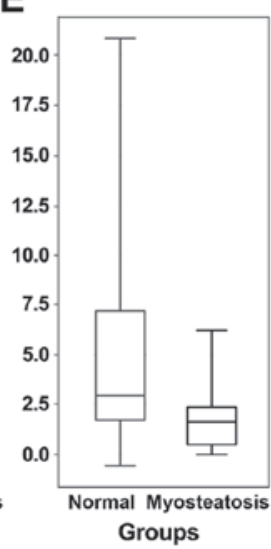

Figure 1. Decision tree analysis models and expression profiles of potentia biomarker peaks. Cluster-peaks implicated in the tree-analysis model using the IMAC30 chip-type (panel A) or the CM10 chip-type (D) stratifying myosteatosis (black) and control (grey) were plotted according to their normalized intensity values (B and $\mathrm{C}$ and $\mathrm{E}, \mathrm{y}$-axis) for peak clusters of $\mathrm{m} / \mathrm{z}$ 2540 (B), 4373 (C) and 2544 (E).

The CM10-based chipset showed 8 peak clusters of interest, of which one was upregulated, 6 downregulated and one unchanged in myosteatosis (e.g., m/z 2,544; Fig. 1E). All of these clustered peaks, which showed statistical significance from both the IMAC30 and CM10 dataset, were further investigated by enrichment of urinary proteins from 14 (IMAC30) and 22 (CM10) samples each, and excision of gel bands after electrophoresis and LC-MS/MS downstream processing, followed by Mascot searching and identification of proteins contained within the samples as described earlier (15). All lead $\mathrm{m} / \mathrm{z}$ clusters have P-values $<0.05$.
Potential markers identified. All potential markers identified by Mascot-SELDI pattern matching are detailed in Table III.

Both the m/z 3,293 and 4,373 from the IMAC30 screen and $\mathrm{m} / \mathrm{z} 3,905$ from the CM10 chipset data could not be matched to observed expression patterns, and $\mathrm{m} / \mathrm{z}$ 19,136 and 22,355 from the CM10 chipset were too far removed from the gel-analysed mass range to assign them to observed protein patterns. Both the $\mathrm{m} / \mathrm{z} 2,540$ and 4,022 clusters from the IMAC30 dataset matched Mannan-binding lectin serine protease 2 precursor (MASP2), and the $\mathrm{m} / \mathrm{z} 2,540$ peak also matched the expression pattern of gelsolin (GSN). The observed expression patterns of both agrin (AGRN) and dipeptidyl-peptidase 1, also known as cathepsin $\mathrm{C}$ (CTSC), matched the $\mathrm{m} / \mathrm{z} 3,934$ peak cluster. The downregulated $\mathrm{m} / \mathrm{z}$ cluster at 2,544 matched to both arylsulfatase A (ARSA) and glial fibrillary acidic protein (GFAP) expression profiles. The downregulated $\mathrm{m} / \mathrm{z} 8,874$ peak cluster expression profile matched Leucine-rich $\alpha$-2-glycoprotein precursor (LRG1). The $\mathrm{m} / \mathrm{z}$ 9,971 cluster was associated with cystatin-B, and the observed histone H2AV (H2AFV) expression correlated with the $\mathrm{m} / \mathrm{z} 7,271$ peak. None of the peak clusters show any significant age-or sex-related expression differences.

The $\mathrm{m} / \mathrm{z}$ distribution of individual peaks within a peak cluster shows the expected pattern of a heterogeneous population of urinary molecular species. However, this does not exclude that some peak clusters may consist of more than one unique protein or peptide.

\section{Discussion}

In the present study, approximately $42 \%$ of patients with uGI cancer were found to have myosteatosis. One potential limitation of this study is that no previously established definition of myosteatosis exists. A value of skeletal muscle density (SMD) 2 SD below the mean in a healthy population was used in a similar manner to definitions for sarcopenia and were selected from values stated in one small study (16). The populations described in other similar studies of skeletal muscle attenuation in healthy individuals are also small, however, and different (although similar) values could be obtained 


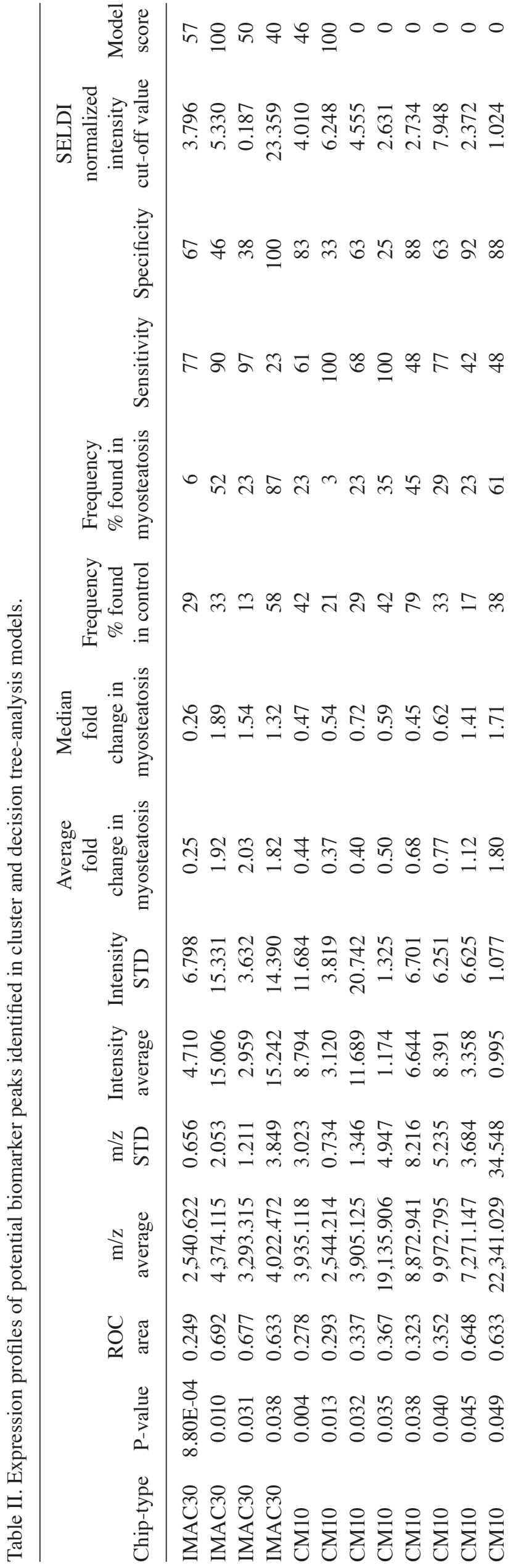

depending on the series chosen $(18,19)$. Skeletal muscle attenuation in the form of HU has recently been demonstrated to be associated with adverse outcome in patients with GI and lung cancer independent of BMI, skeletal muscle mass or weight loss (20). Values obtained for increased risk were $<33$ for individuals with BMI $>25$ or 41 for those with BMI $<25$ (20) and so are not dissimilar to the value of 39.5 chosen for all the participants as a cut-off value for this study. Using this definition, HU values were used to divide the cohort into two groups that had similar characteristics in terms of BMI, weight loss and CRP, suggesting that there was no difference between them in conventional measures of cachexia severity (21). The non-myosteatotic group were slightly older although the difference of seven years is unlikely to be clinically significant.

In this cohort, SELDI-TOF-MS was able to analyse and screen urine from uGI cancer patients for prospective myosteatosis biomarkers and to establish a proteomic fingerprint pattern which potentially can be used in clinical diagnostics. It was found that both IMAC30 and CM10 are useful chip-types for the analysis of human urine (16), and models based on the analysis of 55 samples were generated from cancer patients. The samples were measured on CM10 and IMAC30 chips, and using the tree-analysis method, statistical models were established with overall sensitivities of $97 \%$ (IMAC30) and 77\% (CM10) and specificities of 71\% (IMAC30) and 67\% (CM10) across the entire datasets. Using expression pattern matching, it was possible to assign several proteins identified in urine to the proposed biomarkers.

Cystatin-B is described as a protein that can stimulate cancer cell growth both in vitro and in vivo and recently has been shown to be a potential prognostic marker for lung cancer (22). However, this molecule has no direct known association with skeletal muscle wasting but has been shown to be associated with total fat mass, percentage body fat, abdominal fat and body fat distribution as measured by DEXA in a recent study examining cardiovascular risk factors in children (23).

MASP2 matched the $\mathrm{m} / \mathrm{z} 2,540$ and 4,022 clusters from the IMAC30 dataset; however, $\mathrm{m} / \mathrm{z} 2,540$ was shown to be downregulated, and $\mathrm{m} / \mathrm{z} 4,022$ upregulated. It is a possibility that this could be an indicator that fragmentation states are altered in myosteatosis by for example, lower quantities of other proteases, such as CTSC a lysosomal protease associated with proteolysis after muscle trauma (24), which we found to be associated with the downregulated $\mathrm{m} / \mathrm{z} 3,934$ cluster peak. It has been shown in previous studies to play a role in protein degradation and turnover particularly in the later stages of muscle wasting following disuse (25). Exercise injuries in mouse models have shown upregulation of CTSC in skeletal muscle (26), and our observed downregulation of the associated $\mathrm{m} / \mathrm{z}$ cluster may indicate a dysfunction in muscle repair.

The downregulated $\mathrm{m} / \mathrm{z}$ peak of 2,540 also matched the expression pattern of GSN, which is an actin-filament modulating protein and can be found in smooth and skeletal muscle cells, as well as a secreted form in plasma (27). Reported downregulation of GSN in breast cancer cells, ovarian carcinomas, and melanomas suggests that this molecule acts as a tumour suppressor (28). Underexpression of GSN has been shown to increase inflammation in MS and encephalitits. It has already been reported that recombinant human gelsolin 


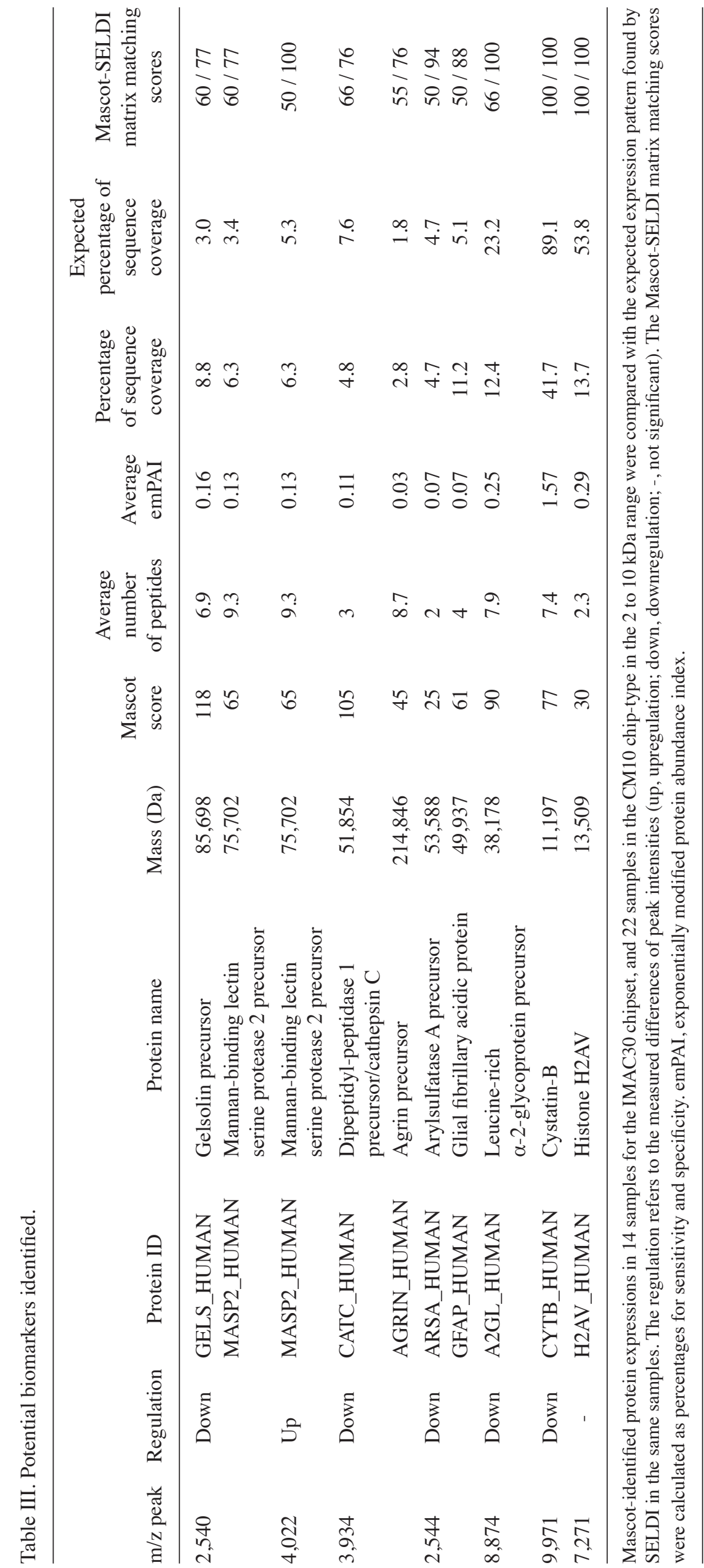


(rhp-GSN) can remit some inflammatory diseases in the animal model, such as burn injury, middle cerebral artery occlusion, and sepsis (29). The association of GSN and apoptosis has been deeply researched. Full-length GSN, and the C-terminal half of GSN are generally considered as having anti-apoptotic function (29).

AGRN, which also matched the same m/z cluster as CTSC. It is a component of the basal lamina on the surface of muscle fibres of the neuromuscular junction, and defects in this gene perturbs the maintenance of the neuromuscular junction (30), whereas an overexpression in a laminin-deficient mouse model increases muscle integrity and regenerative capacity (31). The observed downregulation in the CM10-chipset based model could be related to a dysfunction of muscle regeneration. Conflicting with the downregulation observed herein, however, is the finding that the agrin fragment (CAF) has recently been identified as being elevated in the serum of a series of 73 older volunteers with sarcopenia compared with age matched controls (32). A decrease in CAF has also been associated with improvement of hand grip strength and muscle mass in patients who were undergoing rehabilitation for acute stroke (33). It is possible that this could reflect different mechanisms of wasting in aging and cancer whereby muscle regenerative ability is diminished in cancer but maintained in aging.

The downregulated $\mathrm{m} / \mathrm{z}$ cluster at 2,544 matched to both ARSA and GFAP expression profiles. Mutations in ARSA can cause demyelination and GFAP is associated with central nervous system disorders (27) and chronic neuropathies (32).

The downregulated $\mathrm{m} / \mathrm{z} 8,874$ peak cluster expression profile matched LRG1, which is a secreted protein and has been postulated as a biomarker of ventricular dysfunction and heart failure (34). The reported upregulation associated with heart disease is in contrast with our observed downregulation of this marker, and its role in myosteatosis remains unclear. H2AFV expression correlated with the $\mathrm{m} / \mathrm{z}$ 7,271 peak. Some members of the histone $\mathrm{H} 1$ family have been reported to be involved in cell survival in breast cancer cells and H1.2 was identified as an apoptogenic factor (34). The measured SELDI-MS-based slight upregulation could be an indication of elevated apoptosis in general, and a specific role in muscle function, cancer or fat infiltration remains unclear.

With the exception of AGRN and CTSC, the poor associations of some of these markers with known mechanisms of muscle wasting casts some doubt over the specificity of these results to changes in muscle quality in terms of intramyocellular lipid. One possibility is that some of the markers listed above might also be associated with other factors, such as cancer type. The cohort here included patients with cancers of both oesophagogastric and pancreatic origin and the presence of unrecognised specific markers for these cancer types might skew any interpretation of the results $(35,36)$.

Additional potential limitations are that there may be effects on skeletal muscle HU other than intracellular lipid. A clear association between intramyocellular lipid and Hounsfield units has been demonstrated in healthy individuals (37) and also in patients with cancer cachexia. We have previously shown that the number and size of intramyocellular lipid droplets is increased in the presence of cancer and increases further with weight loss/loss of adipose mass in other body compartments (12). Oedema has been shown to be an additional influence on mean Hounsfield unit of skeletal muscle in patients with cancer that would not be present in healthy volunteers (38). While a number of potential markers indicated such as CTSC, AGRN and arclsulfatase A may be associated with muscle or neuromuscular wasting several of these proteins are non-specific or cancer specific. This may indicate that the models are describing both muscle wasting and additional factors, potentially including cancer progression or nutritional states unrecognised or not assessed in this cohort. The potential influence on mean Hounsfield units in patients with cancer by factors other than intramyocellular lipid also requires to be explored further.

In conclusion, stratification and pattern matching allowed us to search for specific myosteatosis-related biomarkers in the urine of cancer patients using SELDI-TOF mass spectrometry. We observed a downregulation of fragments of CTSC, argin, ARSA and GFAP all of which may play a role muscle wasting. Further investigation of specific assays monitoring expression levels in our proposed biomarkers would help translate our findings into the clinical setting. Investigations using a larger cohort would also confirm their validity.

\section{Acknowledgements}

We thank N.A. Stephens for patient recruitment.

\section{Funding}

Funding of this study was provided by the University of Edinburgh.

\section{Availability of data and materials}

The datasets used and/or analysed during the current study are available from the corresponding author upon reasonable request.

\section{Authors' contributions}

HH carried out the SELDI TOF-MS and contributed to the manuscript. AM undertook the patient assessments and helped with the manuscript. RJES and JM wrote and critically revised the manuscript. AC did the confirmatory mass spectrometry analysis. KCHF and JAR designed the project and JAR gave final approval for the version to be published.

\section{Ethics approval and consent to participate}

Ethics approval was obtained from the Lothian Research Ethics Board and all patients provided written, informed consent.

\section{Consent for publication}

Not applicable.

\section{Competing interests}

The authors have declared no conflict of interest. 


\section{References}

1. Delmonico MJ, Harris TB, Visser M, Park SW, Conroy MB, Velasquez-Mieyer P, Boudreau R, Manini TM, Nevitt M, Newman AB and Goodpaster BH; Health, Aging, and Body: Longitudinal study of muscle strength, quality, and adipose tissue infiltration. Am J Clin Nutr 90: 1579-1585, 2009.

2. Li Y, Xu S, Zhang X, Yi Z and Cichello S: Skeletal intramyocellular lipid metabolism and insulin resistance. Biophys Rev 1: 90-98, 2015.

3. Skipworth RJE, Stewart GD, Dejong CHC, Preston T and Fearon KCH: Pathophysiology of cancer cachexia: Much more than host-tumour interaction? Clin Nutr 26: 667-676, 2007.

4. Dewys WD, Begg C, Lavin PT, Band PR, Bennett JM, Bertino JR, Cohen MH, Douglass HO Jr, Engstrom PF, Ezdinli EZ, et al; Eastern Cooperative Oncology Group: Prognostic effect of weight loss prior to chemotherapy in cancer patients. Am J Med 69: 491-497, 1980.

5. Fearon KCH, Voss AC and Hustead DS; Cancer Cachexia Study Group: Definition of cancer cachexia: Effect of weight loss reduced food intake, and systemic inflammation on functional status and prognosis. Am J Clin Nutr 83: 1345-1350, 2006.

6. Lieffers JR, Bathe OF, Fassbender $\mathrm{K}$, Winget $\mathrm{M}$ and Baracos VE: Sarcopenia is associated with postoperative infection and delayed recovery from colorectal cancer resection surgery. $\mathrm{Br} \mathrm{J}$ Cancer 107: 931-936, 2012.

7. Ding Q, Mracek T, Gonzalez-Muniesa P, Kos K, Wilding J, Trayhurn $\mathrm{P}$ and Bing C: Identification of macrophage inhibitory cytokine-1 in adipose tissue and its secretion as an adipokine by human adipocytes. Endocrinology 150: 1688-1696, 2009.

8. Russell ST and Tisdale MJ: The role of glucocorticoids in the induction of zinc-alpha2-glycoprotein expression in adipose tissue in cancer cachexia. Br J Cancer 92: 876-881, 2005.

9. Mracek T, Stephens NA, Gao D, Bao Y, Ross JA, Rydén M, Arner P, Trayhurn P, Fearon KC and Bing C: Enhanced ZAG production by subcutaneous adipose tissue is linked to weight loss in gastrointestinal cancer patients. Br J Cancer 104: 441-447, 2011.

10. Das SK, Eder S, Schauer S, Diwoky C, Temmel H, Guertl B, Gorkiewicz G, Tamilarasan KP, Kumari P, Trauner M, et al: Adipose triglyceride lipase contributes to cancer-associated cachexia. Science 333: 233-238, 2011.

11. Gray C, MacGillivray TJ, Eeley C, Stephens NA, Beggs I, Fearon $\mathrm{KCH}$ and Greig CA: Magnetic resonance imaging with k-means clustering objectively measures whole muscle volume compartments in sarcopenia/cancer cachexia. Clin Nutr 30 106-111, 2011.

12. Stephens NA, Skipworth RJE, Macdonald AJ, Greig CA, Ross JA and Fearon $\mathrm{KCH}$ : Intramyocellular lipid droplets increase with progression of cachexia in cancer patients. J Cachexia Sarcopenia Muscle 2: 111-117, 2011.

13. Mueller TC, Bachmann J, Prokopchuk O, Friess $\mathrm{H}$ and Martignoni ME: Molecular pathways leading to loss of skeletal muscle mass in cancer cachexia - can findings from animal models be translated to humans? BMC Cancer 16: 75, 2016.

14. Fearon KCH and Baracos VE: Cachexia in pancreatic cancer: New treatment options and measures of success. HPB 12: 323-324, 2010.

15. Skipworth RJE, Stewart GD, Bhana M, Christie J, Sturgeon CM, Guttridge DC, Cronshaw AD, Fearon KCH and Ross JA: Mass spectrometric detection of candidate protein biomarkers of cancer cachexia in human urine. Int J Oncol 36: 973-982, 2010.

16. Husi H, Stephens N, Cronshaw A, MacDonald A, Gallagher I, Greig C, Fearon $\mathrm{KCH}$ and Ross JA: Proteomic analysis of urinary upper gastrointestinal cancer markers. Proteomics Clin Appl 5: 289-299, 2011

17. Prado CM, Lieffers JR, McCargar LJ, Reiman T, Sawyer MB, Martin L and Baracos VE: Prevalence and clinical implications of sarcopenic obesity in patients with solid tumours of the respiratory and gastrointestinal tracts: A population-based study. Lancet Oncol 9: 629-635, 2008

18. Goodpaster BH, Thaete FL and Kelley DE: Composition of skeletal muscle evaluated with computed tomography. Ann N Y Acad Sci 904: 18-24, 2000

19. Goodpaster BH, Carlson CL, Visser M, Kelley DE, Scherzinger A, Harris TB, Stamm E and Newman AB: Attenuation of skeletal muscle and strength in the elderly: The Health ABC Study. J Appl Physiol 1985 90: 2157-2165, 2001.
20. Martin L, Birdsell L, Macdonald N, Reiman T, Clandinin MT, McCargar LJ, Murphy R, Ghosh S, Sawyer MB and Baracos VE: Cancer cachexia in the age of obesity: Skeletal muscle depletion is a powerful prognostic factor, independent of body mass index. J Clin Oncol 31: 1539-1547, 2013.

21. Fearon K, Strasser F, Anker SD, Bosaeus I, Bruera E, Fainsinger RL, Jatoi A, Loprinzi C, MacDonald N, Mantovani G, et al: Definition and classification of cancer cachexia: An international consensus. Lancet Oncol 12: 489-495, 2011.

22. Hosokawa M, Kashiwaya K, Eguchi H, Ohigashi H, Ishikawa O, Furihata M, Shinomura Y, Imai K, Nakamura Y and Nakagawa H: Over-expression of cysteine proteinase inhibitor cystatin 6 promotes pancreatic cancer growth. Cancer Sci 99: 1626-1632, 2008.

23. Dencker M, Tanha T, Karlsson MK, Wollmer P, Andersen LB and Thorsson O: Cystatin B, cathepsin L and D related to surrogate markers for cardiovascular disease in children. PLoS One 12: e0187494, 2017.

24. Farges MC, Balcerzak D, Fisher BD, Attaix D, Béchet D, Ferrara M and Baracos VE: Increased muscle proteolysis after local trauma mainly reflects macrophage-associated lysosomal proteolysis. Am J Physiol Endocrinol Metab 282: E326-E335, 2002.

25. Stevenson EJ, Giresi PG, Koncarevic A and Kandarian SC: Global analysis of gene expression patterns during disuse atrophy in rat skeletal muscle. J Physiol 551: 33-48, 2003.

26. Salminen A and Kihlström M: Lysosomal changes in mouse skeletal muscle during the repair of exercise injuries. Muscle Nerve 8: 269-279, 1985.

27. Wen D, Corina K, Chow EP, Miller S, Janmey PA and Pepinsky RB: The plasma and cytoplasmic forms of human gelsolin differ in disulfide structure. Biochemistry 35: 9700-9709, 1996.

28. Rao J and Li N: Microfilament actin remodeling as a potential target for cancer drug development. Curr Cancer Drug Targets 4 : 345-354, 2004.

29. Gao J, Qin Z, Guan X, Guo J, Wang H and Liu S: Overexpression of GSN could decrease inflammation and apoptosis in EAE and may enhance vitamin D therapy on EAE/MS. Sci Rep 7: 604, 2017.

30. Bentzinger CF, Barzaghi P,Lin S and Ruegg MA: Overexpression of mini-agrin in skeletal muscle increases muscle integrity and regenerative capacity in laminin-alpha2-deficient mice. FASEB J 19 934-942, 2005.

31. Hettwer S, Dahinden P, Kucsera S, Farina C, Ahmed S, Fariello R, Drey M, Sieber CC and Vrijbloed JW: Elevated levels of a C-terminal agrin fragment identifies a new subset of sarcopenia patients. Exp Gerontol 48: 69-75, 2013.

32. Brenner M, Johnson AB, Boespflug-Tanguy O, Rodriguez D, Goldman JE and Messing A: Mutations in GFAP, encoding glial fibrillary acidic protein, are associated with Alexander disease. Nat Genet 27: 117-120, 2001.

33. Scherbakov N, Knops M, Ebner N, Valentova M, Sandek A, Grittner U, Dahinden P, Hettwer S, Schefold JC, von Haehling S, et al: Evaluation of C-terminal agrin fragment as a marker of muscle wasting in patients after acute stroke during early rehabilitation. J Cachexia Sarcopenia Muscle 7: 60-67, 2016.

34. Watson CJ, Ledwidge MT, Phelan D, Collier P, Byrne JC, Dunn MJ, McDonald KM and Baugh JA: Proteomic analysis of coronary sinus serum reveals leucine-rich $\alpha 2$-glycoprotein as a novel biomarker of ventricular dysfunction and heart failure. Circ Heart Fail 4: 188-197, 2011.

35. Huzé C, Bauché S, Richard P, Chevessier F, Goillot E, Gaudon K, et al: Identification of an agrin mutation that causes congenital myasthenia and affects synapse function. Am J Hum Genet 85: 155-167, 2009.

36. Bentzinger CF, Barzaghi P, Lin S and Ruegg MA: Overexpression of mini-agrin in skeletal muscle increases muscle integrity and regenerative capacity in laminin-alpha2-deficient mice. FASEB J 19: 934-942, 2005.

37. Goodpaster BH, Kelley DE, Thaete FL, He J and Ross R: Skeletal muscle attenuation determined by computed tomography is associated with skeletal muscle lipid content. J Appl Physiol (1985) 89: 104-110, 2000

38. Ramage MI, Johns N, Deans CD, Ross JA, Preston T, Skipworth RJ, Jacobi C and Fearon KC: The relationship between muscle protein content and CT-derived muscle radio-density in patients with upper GI cancer. Clin Nutr 37: 752-754, 2018. 